\title{
Observations and modeling of forward and reflected chorus waves captured by THEMIS
}

\author{
O. Agapitov ${ }^{1,2}$, V. Krasnoselskikh ${ }^{1}$, Yu. Zaliznyak ${ }^{1,3}$, V. Angelopoulos ${ }^{4}$, O. Le Contel ${ }^{5}$, and G. Rolland ${ }^{6}$ \\ ${ }^{1}$ LPCE/CNRS-University of Orleans, Orleans, France \\ ${ }^{2}$ National Taras Shevchenko University of Kyiv, Kyiv, Ukraine \\ ${ }^{3}$ Institute for Nuclear Research, Kyiv, Ukraine \\ ${ }^{4}$ Institute of Geophyiscs and Planetary Physics University of California, Los Angeles (UCLA), USA \\ ${ }^{5}$ Laboratoire de Physique des Plasmas, St Maur-des-Fosses, France \\ ${ }^{6} \mathrm{CNES}$, France
}

Received: 29 December 2010 - Revised: 22 February 2011 - Accepted: 23 February 2011 - Published: 11 March 2011

\begin{abstract}
Discrete ELF/VLF chorus emissions are the most intense electromagnetic plasma waves observed in the radiation belts of the Earth's magnetosphere. Chorus emissions, whistler-mode wave packets propagating roughly along magnetic field lines from a well-localized source in the vicinity of the magnetic equator to polar regions, can be reflected at low altitudes. After reflection, wave packets can return to the equatorial plane region. Understanding of whistler wave propagation and reflection is critical to a correct description of wave-particle interaction in the radiation belts. We focus on properties of reflected chorus emissions observed by the THEMIS (Time History of Events and Macroscale Interactions During Substorms) spacecraft Search Coil Magnetometer (SCM) and Electric Field Instrument (EFI) at ELF/VLF frequencies up to $4 \mathrm{kHz}$ at $L \geq 8$. We determine the direction of the Poynting flux and wave vector distribution for forward and reflected chorus waves. Although both types of chorus waves were detected near the magnetic equator and have similar, discrete structure and rising tones, reflected waves are attenuated by a factor of 10-30 and have $10 \%$ higher frequency than concurrently-observed forward waves. Modeling of wave propagation and reflection using geometrical optics ray-tracing allowed us to determine the chorus source region location and explain observed propagation characteristics. We find that reflected wave attenuation at a certain spatial region is caused by divergence of the ray paths of these non-ducted emissions, and that the frequency shift is caused by generation of the reflected waves at lower L-shells where the local equatorial gyrofrequency is larger.
\end{abstract}

Keywords. Electromagnetics (Wave propagation)

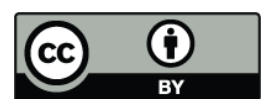

Correspondence to: O. Agapitov (agapit@univ.kiev.ua)

\section{Introduction}

The most intense electromagnetic plasma waves observed in Earth's radiation belts and outer magnetosphere, discrete ELF/VLF chorus emissions are characterized by rising and falling tones in the few hundred to several thousand Hertz frequency range (Burton and Holzer, 1974; Meredith et al., 2001; Gurnett and O'Brien, 1964; Tsurutani and Smith, 1974, 1977; Anderson and Maeda, 1977; Haque et al., 2010; Koons and Roeder, 1990). See also reviews by Omura et al. (1991) and Sazhin and Hayakawa (1992) and references therein. Critical to radiation belt dynamics, these emissions have been studied intensively because they play a crucial role in the acceleration of energetic electrons at the outer radiation belt (Horne et al, 2005). Typically ELF/VLF chorus emissions are observed near the magnetic equatorial plane in the dawn and dayside outer magnetosphere (Omura et al., 1991). They have attracted special attention recently because they were observed as waveforms, allowing determination of their wave normal vector distributions. Burton and Holzer (1974) were the first who determined chorus normal vector distributions using the search coil magnetometer aboard OGO5, near the equator, at geosynchronous altitude. Chorus wave normal directions at $\leq 17^{\circ}$ magnetic latitudes and L-shells $~ 7.6$ were later studied by Hayakawa et al. (1990). Analysis of wave normals and Poynting fluxes for separate emission elements has shown that the emissions are generated in proximity to the geomagnetic equator and propagate to higher latitudes in a non-ducted whistler mode (Burton and Holzer, 1974; Hayakawa et al., 1990; Yagitani et al., 1999). Chorus emissions are usually observed in the Earth's dawn sector between 23:00 and 13:00 MLT (Tsurutani and Smith, 1974). These emissions, which propagate in the whistler mode, consist of two broad frequency bands on either side of the local equatorial gyrofrequency $f_{\text {ce }}$ at the

Published by Copernicus Publications on behalf of the European Geosciences Union. 
geomagnetic equator along the magnetic field line on which the waves are observed (Tsurutani and Smith, 1974, 1977). If present, the upper band exists in the $f / f_{\mathrm{ce}} \approx 0.5-0.75$ frequency range and contains discrete chorus elements rising at a few $\mathrm{kHz} / \mathrm{s}$. The lower band exists in the $f / f_{\text {ce }} \approx 0.1-0.5$ frequency range and contains both elements rising at a few $\mathrm{kHz} / \mathrm{s}$ and diffuse elements. In the inner magnetosphere, $\mathrm{L}$ shell is $\sim 2-6$, wave generation onset has been shown to be associated with substorm electron injections (Goldstein and Tsurutani, 1984). Chorus in the radiation belts is believed to be generated through electron cyclotron instability when the distribution of energetic electrons in the 5 to $150 \mathrm{keV}$ range is strongly anisotropic (Helliwell, 1967; Burton, 1976; Trakhtengerts, 1999). This has been shown to be the case in recent studies of such waves during electron injections. Chorus waves in the outer dayside region have received further attention recently, especially as the THEMIS mission was able to extend previous observations beyond $L \sim 7$ to $L \sim 13$. $\mathrm{Li}$ et al. (2009b) showed that chorus waves are present at $L>7$ up to just inside the magnetopause, even during quiet times. These dayside outer magnetosphere chorus emissions were observed with non-thermal particles by the Geotail spacecraft (Yagitani et al., 1999), indicating that the particle anisotropy was too small to generate chorus emissions by linear cyclotron anisotropy. Li et al. (2010), however, analyzed particle anisotropies, flux and equatorial density distribution observed by THEMIS spacecraft to determine the anisotropy at the chorus resonant energies and found good agreement of the unstable modes with the observed distribution of chorus waves in the ( $\mathrm{Li}$ et al., 2009a) study. Dayside quiet-time chorus cannot be explained in the absence of injections, however, and remains an area of active study. Other chorus generation occur in the local minimum magnetic field regions near the dayside magnetopause on the magnetic latitude near $\pm\left(40^{\circ}-50^{\circ}\right)$ (Tsurutani and Smith, 1977). The spatial and temporal dependencies of high-latitude chorus parameters are considerably different from those of the chorus generated near the magnetic equator. The frequency range of the highlatitude chorus is similar to chorus waves generated near the magnetic equator at $L>10$ but their spectral power distribution exhibits two maxima: $(0-0.15) f_{\text {ce }}$ and $(0.25-0.30) f_{\text {ce }}$. These emissions are mainly detected within $1-2 R_{\mathrm{E}}$ from the magnetopause (Tsurutani and Smith, 1977).

Another emission typically observed in the radiation belt region is hiss, which has slightly lower frequencies and different structural characteristics than chorus. Chorus and hiss often co-exist; both are frequently observed simultaneously by satellites in the outer magnetosphere. Bortnik et al. (2009) showed that plasmaspheric hiss can be observed simultaneously with dayside or dawn chorus. Using ray-tracing techniques, they explained hiss generation as plasmaspheric penetration of lower-band chorus from multiple L-shells beyond the plasmapause and multiple MLTs. Modeling of ray traces and amplitudes agreed with THEMIS observations of such waves from high-resolution waveform captures during fortu- itous long-range spacecraft conjunctions. Therefore, chorus wave propagation characteristics are critical in determining the location and amplitude of plasmaspheric hiss.

Before the Cluster mission, observations of ELF/VLF chorus emissions were mainly made by single spacecraft, such as ISEE 1 and ISEE 2, which observed many such events (Gurnett et al., 1979). Recent Poynting flux and polarization measurements aboard Cluster spacecraft confirmed that the chorus source is located close to the equatorial plane (Parrot et al., 2004; Santolik et al., 2005). Measurements around the magnetic equator demonstrate the change in sign of the parallel component of the Poynting vector when the satellites cross the equator region. Poynting vector flux analysis indicates that the central position of the chorus source fluctuates along the background magnetic field within $1000-2000 \mathrm{~km}$ of the geomagnetic equatorial plane for minutes. In studies of chorus emission generation mechanisms, Helliwell (1967) and Trakhtengerts (1999) gave theoretical estimates of the scale size of the wave generation region. Attempts to estimate the scale size experimentally were made using coordinated CLUSTER (Santolik et al., 2005; Agapitov et al., 2011) and more recently THEMIS (Agapitov et al., 2010) observations in the radiation belt region.

Although the magnetospheric reflection of whistler chorus is discussed and simulated in a number of papers, such as Helliwell (1967) and Burton and Holzer (1974), experimental confirmation is scarce. Continuous increase in the angle between the wave vector and background magnetic field for several cases of reflected chorus in the outer magnetosphere, based on Ogo 5 measurements, was shown in Burton and Holzer (1974). In Parrot et al. (2004) the Poynting vector and wave normal directions of chorus waves were analyzed using Cluster STAFF-SA measurements of spectral matrices with a $4 \mathrm{~s}$ time resolution. Different Cluster spacecraft observed waves propagating from the geomagnetic equator region and reflected waves that reached a lower hybrid resonance reflection at low altitudes and returned to the equator at another location with a lower intensity. Parrot et al. (2004) corroborated this interpretation using ray tracing analysis. Bortnik et al. (2009) demonstrated wave reflection, refraction and resultant inward radial propagation across L-shells and MLT over several $R_{\mathrm{E}}$. The agreement between models and THEMIS data in the above study Bortnik et al. (2009) encourages use of the ray tracing technique to determine evolution and consequences of chorus waves outside the plasmasphere.

In this paper direct and reflected chorus emission propagation characteristics are considered. The data set chosen for the study was recorded by the THA THEMIS spacecraft on 26 July 2008 near 14:00 UT in the outer magnetosphere. The data make it possible to compare the properties of forward and reflected chorus waves generated by the same source. We use high-resolution magnetic and electric field waveform measurements recorded by the SCM and EFI instruments in burst mode to analyze the discrete internal structure of 
direct and reflected chorus waves. Propagation of whistler wave packets was also simulated in the framework of raytracing with realistic magnetospheric plasma particle density and magnetic field distributions.

This paper is organized as follows: in Sect. 2 we describe THEMIS SCM and EFI data. Results of THEMIS data processing for direct and reflected chorus events are shown in Sect. 3. In Sect. 4, implementation of the ray-tracing technique for whistler wave propagation in the magnetosphere is described, and the technique is applied to simulate directlypropagating and reflected whistler emissions captured aboard THEMIS satellites. Conclusions follow in Sect. 5.

\section{Data description and processing technique}

THEMIS (Time History of Events and Macroscale Interactions During Substorms) consists of five identicallyinstrumented spacecraft (THA, THB, THC, THD, and THE), launched on 17 February 2007. The main goal of this mission is to conduct multi-point investigations of substorm phenomena in the tail of the terrestrial magnetosphere (Sibeck and Angelopoulos, 2008). For the current study, Search Coil Magnetometer (SCM) observations (Le Contel et al., 2008) and plasma measurements of the Electric Field Instrument (EFI) (Bonnell et al., 2008) were analyzed. The three search coil antennas cover the same bandwidth, from $0.1 \mathrm{~Hz}$ to $4 \mathrm{kHz}$, in the ULF/ELF frequency range. The electric field components are measured directly in the same frequency range. Three components of magnetic field waveform (scw files) and three components of electric field waveform (efw files) captured in the wave burst mode (on-board trigger) with $8192 \mathrm{samp} / \mathrm{s}$ were used.

In this study the Means method (Means, 1972), minimum variance analysis of the spectral variation matrix, and singular value decomposition (SVD) (Santolik et al., 2003) are used to determine the wave normal vector $\boldsymbol{k}$. The Means method involves computation of a spectral matrix that consists of power and cross-power spectra from the three magnetic components. Although this method has an inherent $180^{\circ}$ ambiguity in the wave normal direction, this ambiguity can be removed if the Poynting vector $S$ is known. Since the wave normal vector must have a component in the direction of the energy flow, a scalar product $(\boldsymbol{S} \cdot \boldsymbol{k})$ should be positive.

The Poynting vector is calculated directly: $\boldsymbol{S}=$ $(1 / 2) \operatorname{Re}\left(\boldsymbol{E}(f) \times \boldsymbol{B}(f)^{*}\right)$, where ${ }^{*}$ represents the complex conjugate, Re stands for the real part, and $\boldsymbol{E}(f)$ and $\boldsymbol{B}(f)$ are the Fourier transforms of the electric and magnetic field waveforms, respectively.

\section{Direct and reflected whistler chorus: the data}

The well-studied properties of lightning-generated whistlers make them very useful for checking our implementation of the wave normal and Poynting vector reconstruction tools. A lightning stroke produces a burst of broadband electromagnetic radiation part of which can escape to the plasmasphere and travel along closed field lines from one hemisphere to the other in the form of a right-hand-polarized whistler wave. The ducted propagation of the atmospherically generated whistlers allow their multiple observation in the same spatial location (Shklyar et al., 2004). The characteristic spectrum of a whistler is a tone rapidly decreasing in frequency over a few seconds. On 11 July 2008 from 12:25 to 13:09 UT, high amplitude, lightning-generated whistlers were detected by the SCM and EFI of THD. THD is at $R_{\mathrm{GSE}}=[1.1,2.9,0.17]$, $R_{\mathrm{SM}}=[2.96,-0.76,0.18]$, MLat $=2.6-4$, Dst $=1-3 \mathrm{nT}$, the electron gyrofrequency $f_{\text {ce }}=24660 \mathrm{~Hz}$. Detailed timefrequency diagrams of magnetic field and electric field perturbations and the Poynting vector flux direction and magnitude are shown in Fig. 1. A sequence of whistler wave packets propagating alternate poleward and to the equator with typical falling frequency spectra is clearly seen. The Poynting flux demonstrates the change in propagation direction after reflection. Observed events show a rapid decrease in the reflected signal amplitude below $500-600 \mathrm{~Hz}$, which is in a good agreement with the estimated value of the local plasma lower hybrid frequency $f_{\mathrm{LH}}$ as $1 / 40$ of $f_{\text {ce }}$, $f_{\mathrm{LH}} \approx 600 \mathrm{~Hz}$. Detection of the same whistler wave packet in the same spatial region after reflection indicates that ducted whistler wave propagation was observed. This type of propagation is typical for whistler waves generated in the atmosphere (Helliwell, 1967). Waves propagate in a plasma density inhomogeneity duct along magnetic field lines. Thus, atmospherically-generated whistler waves can be detected several times on the same magnetic shell after multiple reflections. Reflection of ducted waves can occur at magnetospheric as well as ionospheric altitudes. The reflected whistler waves show conservation of energy with temporal spreading due to wave dispersion.

Agreement of observed properties of lightning-generated whistlers with theoretically predicted (Helliwell, 1967) and numerically-simulated (Shklyar et al., 2004) properties, such as conservation of energy during propagation and reflection, temporal spreading according to the whistler wave dispersion, and repeated observation of the whistler waves at the same L-shell after reflection confirms our implementation of the wave normal and Poynting vector reconstruction technique.

Next we study the other type of whistler waves in the Earth's magnetosphere - magnetospherically-generated chorus emissions, which usually propagate as non-ducted modes. Using electric and magnetic field fluctuation measurements, we determine the direction and magnitude of the Poynting flux and wave vector distribution for direct and reflected chorus waves. Although reflected chorus emissions observed in the vicinity of the magnetic equator have a discrete structure roughly similar to that of the direct chorus, the amplitude of the reflected signal is significantly (ten to thirty times) smaller. The case study of one selected event is 

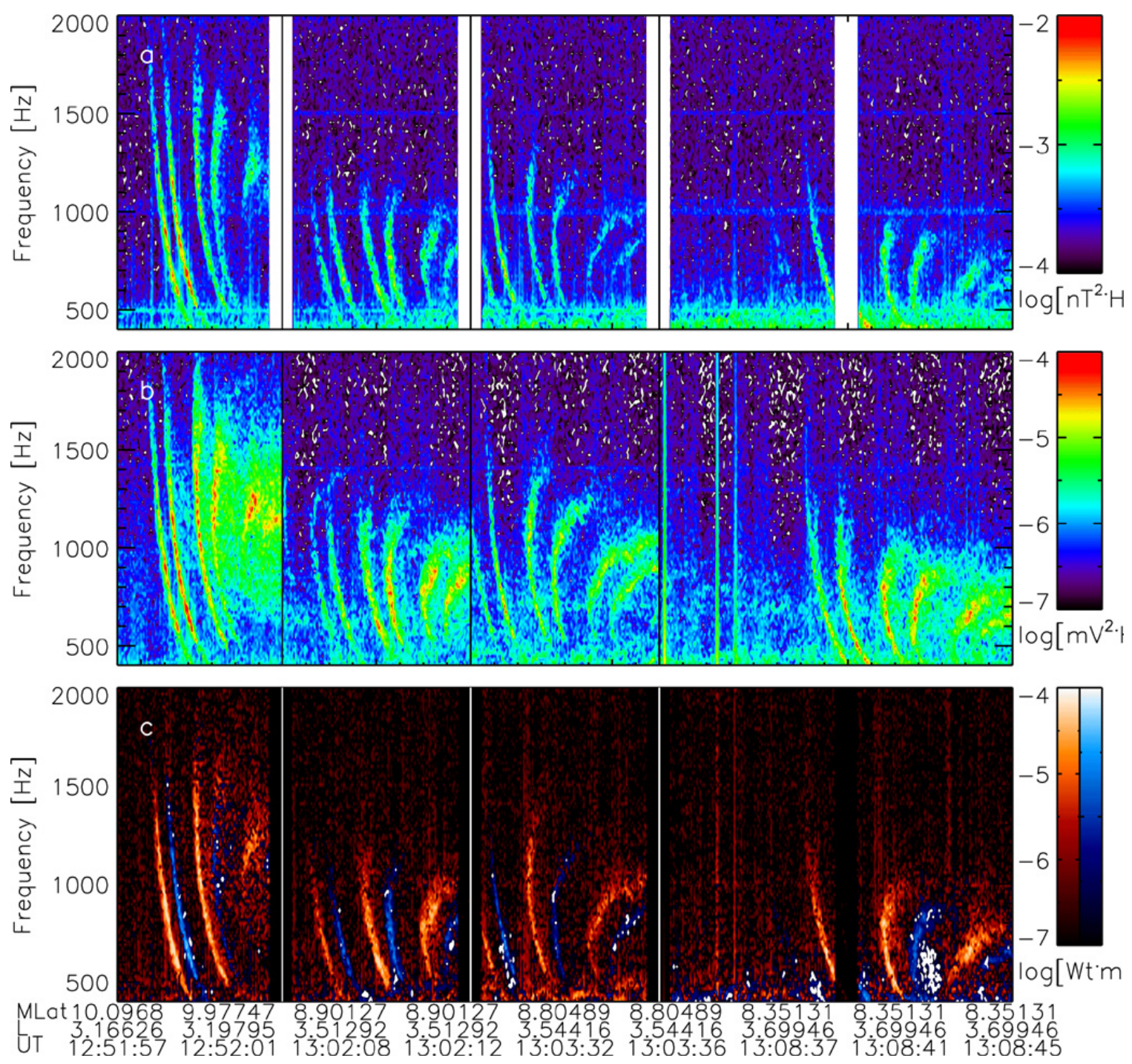

Fig. 1. Time-frequency spectrum of multiple whistler events observed by the SCM/SCW (a) and EFI/EFW (b) of THD on 11 June 2008 . In panel (c) the Poynting vector direction is shown (red - from the equator, blue - to the equator). Spectral power is in logarithmic scale.

presented below along with a detailed numerical reconstruction using ray-tracing techniques. Well-resolved measurements of the three magnetic field components and two electric field components by instruments on THEMIS spacecraft allow us to study the forward discrete chorus wave packets and, simultaneously, the reflected chorus propagated back to the magnetic equator plane. The event chosen is particularly interesting because amplitude and frequency characteristics of the direct waves can be compared with those of reflected waves generated by the same source.

Figure 2 presents detailed time-frequency diagrams of magnetic field and electric field perturbations and the Poynting flux vector recorded aboard THA on 26 July 2008 when it was close to local magnetic noon and to the geomagnetic equator plane, $R_{\mathrm{SM}}=[8.4,0.15,0.42], \mathrm{MLat}=2.8^{\circ}-2.9^{\circ}$, $\mathrm{Kp}=0$, Dst $=-8 \mathrm{nT}$. The local electron gyrofrequency is $f_{\text {ce }}=1750 \mathrm{~Hz}$. Discrete chorus emissions are clearly seen in the two frequency diapasons $\left(0.15-0.45 f_{\text {ce }}\right.$ and $0.5-0.6$ $f_{\text {ce }}$ ) typical for chorus waves generated in the vicinity of the magnetic equator. The projection of the Poynting vector on the local magnetic field is shown in the lower panel of Fig. 2. The Poyntig flux direction along the local magnetic field (poleward) is marked with red color gamma and the opposite direction (equatorward) is marked with blue.

High-amplitude discrete chorus elements are followed by lower amplitude elements with a frequency range from 0.2 $f_{\text {ce }}$ to $0.5 f_{\text {ce }}$ and different frequency rise speed. These elements can be clearly seen in the Poynting vector flux panel as elements with the Poynting vector directed equatorward. Such chorus waves can be generated on high-latitudes near the magnetopause (Tsurutani and Smith, 1977). Usually the high-latitude chorus waves are observed on distances 

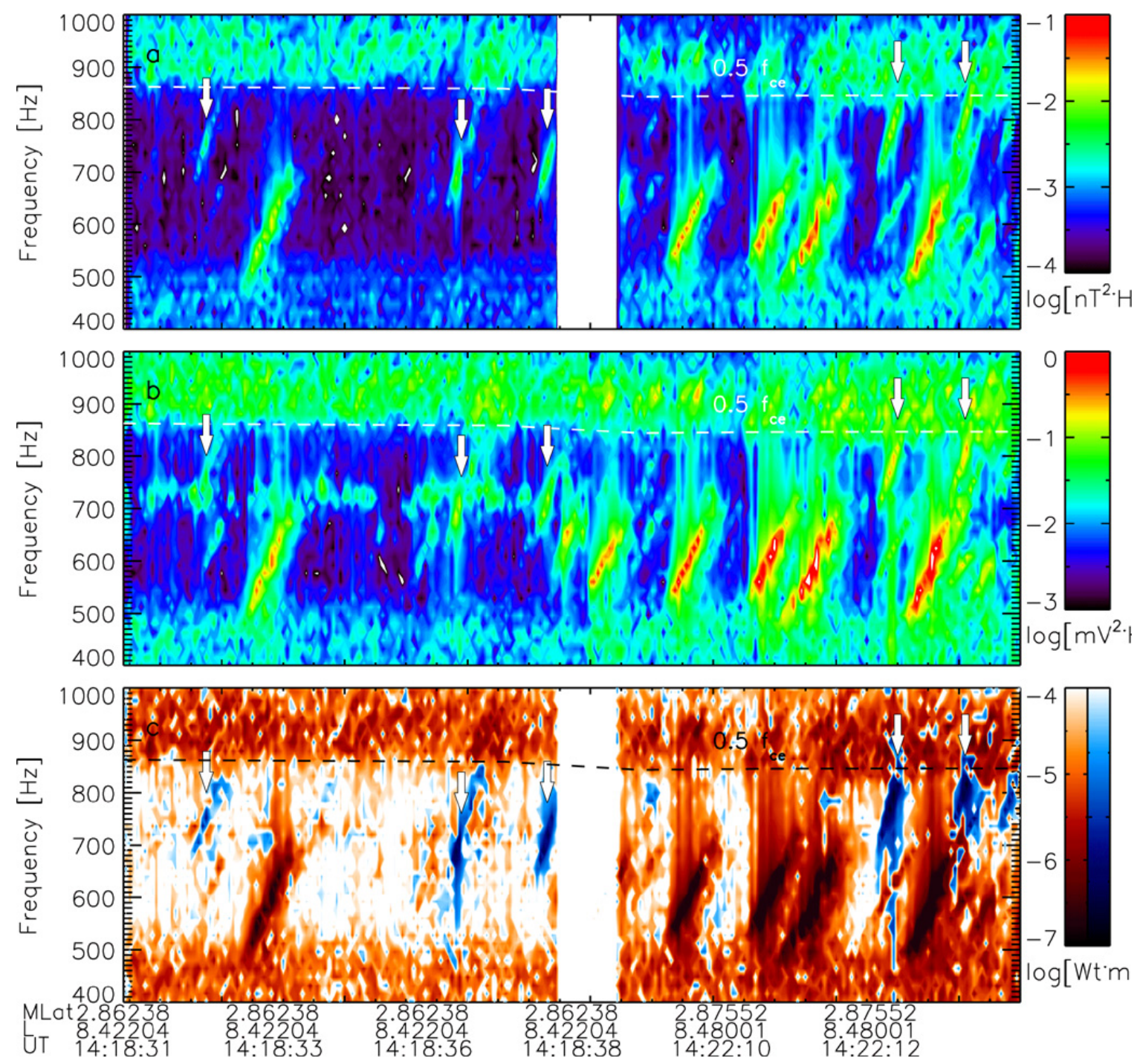

Fig. 2. Detailed time-frequency power spectrograms of magnetic (top) and electric (middle) field fluctuations near the source region recorded by the SCM (a) and EFI (b) instruments aboard THA on 26 July 2008. Frequency-time dependence of the Poynting vector direction of forward and reflected chorus observed by the SCM/SCW (c). The field aligned waves elements are marked with red color and the elements propagated in opposite direction are marked with blue color. Spectral power is in logarithmic scale. The reflected chorus elements are marked with white arrows.

$1-2 R_{\mathrm{E}}$ from the magnetopause (curren position is about $12-$ $13 R_{\mathrm{E}}$ ) (Tsurutani and Smith, 1977). The spectral power frequency profile is close to observed one for the equator generated emissions. Thus we speculate that the observed lowamplitude wave packets are generated in the vicinity of the magnetic equator, propagate poleward, magnetospherically reflected and then return to the equator. This thesis will be affirmed in Sect. 4 with ray-tracing technique.

The frequency shift with respect to chorus propagating from the magnetic equator observed by THA shows that the reflected chorus packets were generated closer to the Earth in the vicinity of the geomagnetic equator. The directions of the $\boldsymbol{k}$ vectors and Poynting vectors are shown in Figs. 3 and 4, respectively. The direct chorus elements propagate roughly along the local magnetic field (shown in Figs. 3 and 4 by the red cross) which is usual for the generation region. The Poynting vector direction of the reflected chorus elements is close to the local magnetic field, but the $\boldsymbol{k}$ vector is inclined nearly $35^{\circ}-40^{\circ}$ with respect to magnetic field but in opposite direction. The amplitude ratio for forward and reflected waves varies from 10 to 30 for the magnetic field perturbation and from 10 to 30 for the electric field (Fig. 5). Estimation of the Poynting flux ratio gives a value from 100 to 400. Assuming that the initial amplitude of the reflected chorus was of the same order with the observed direct waves (Burtis and Helliwell, 1969) the decrease in the amplitude of the reflected 

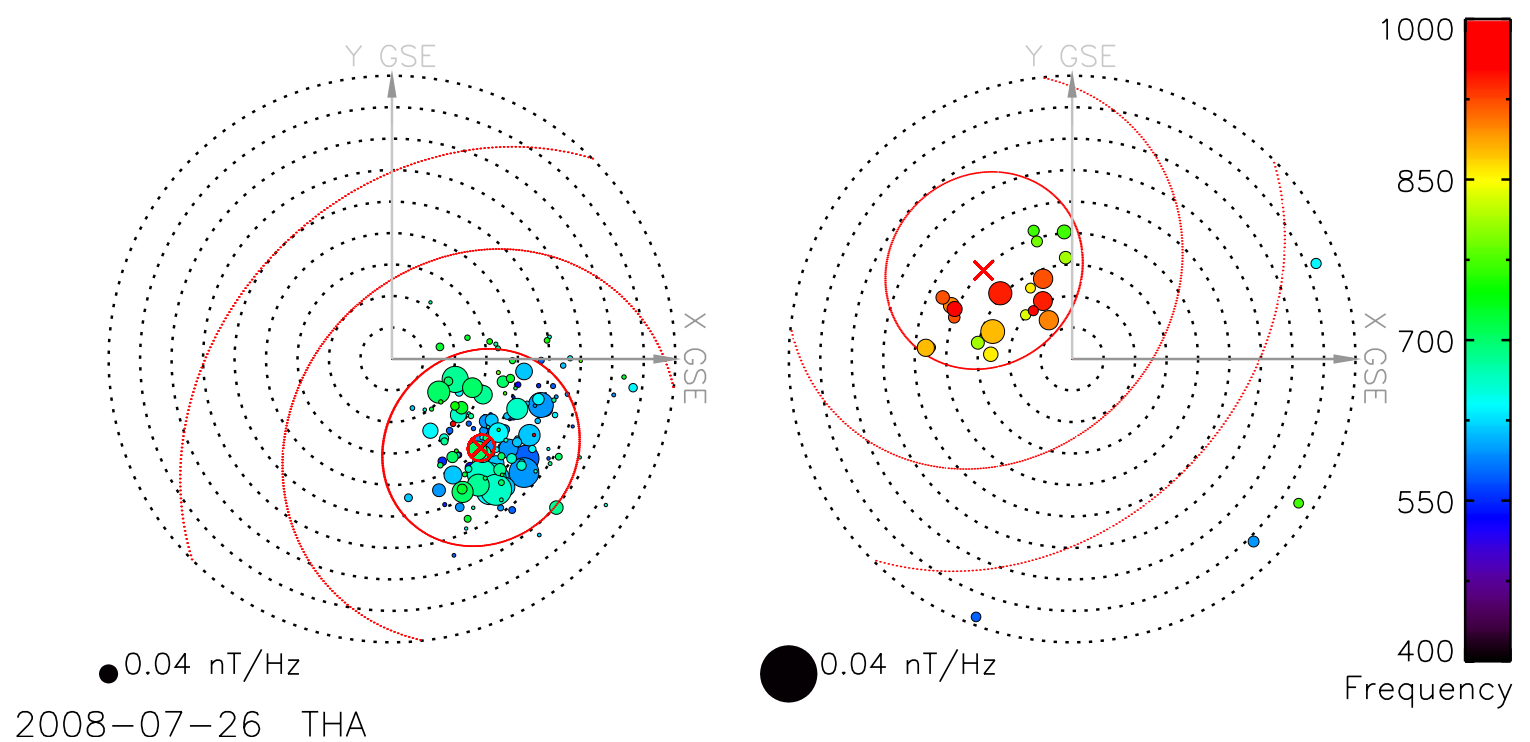

Fig. 3. The $\boldsymbol{k}$-vector directions of the wave packets. The Sun is to the right, and the polar axis is along $Z_{\mathrm{GSM}}$. The background magnetic field direction is indicated by the red cross in the circle. The opposite direction is indicated by the red cross (left). The colors of the circles show the wave frequency according to color bar; the radius of the circles is proportional to the wave magnetic field amplitude shown by black circle.
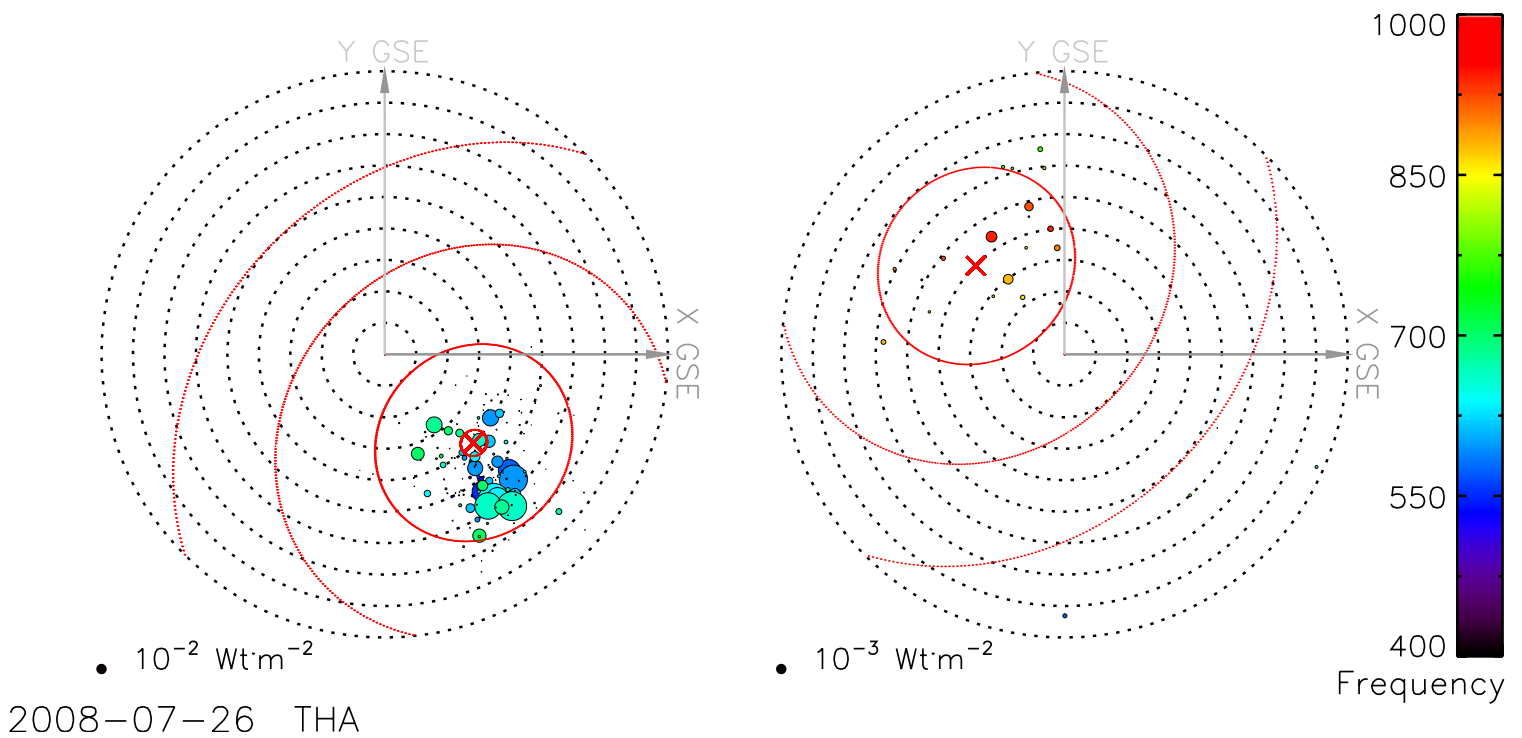

Fig. 4. The Poynting vector directions of the wave packets. The Sun is to the right, and the polar axis is along $Z_{\mathrm{GSM}}$. The background magnetic field direction is indicated by the red cross in the circle. The opposite direction is indicated by the red cross (right). The colors of the circles show the wave frequency according to color bar; the radius of the circles is proportional to the Poynting flux magnitude shown by black circle.

signal could be due to geometrical effects of chorus wave propagation and spreading of the pulse by dispersion. Chorus waves can be generated in the extended radiation source in the similar frequency range relative to the local electron gyrofrequency. In this case the observed wave amplitudes on similar frequency range relative to gyrofrequency are close (Burtis and Helliwell, 1969).
To test this hypothesis, we model chorus wave propagation and reflection using a ray-tracing technique. As we see below, this technique allows us to reconstruct the chorus source region and explain its observed frequency shifts and propagation characteristics. 

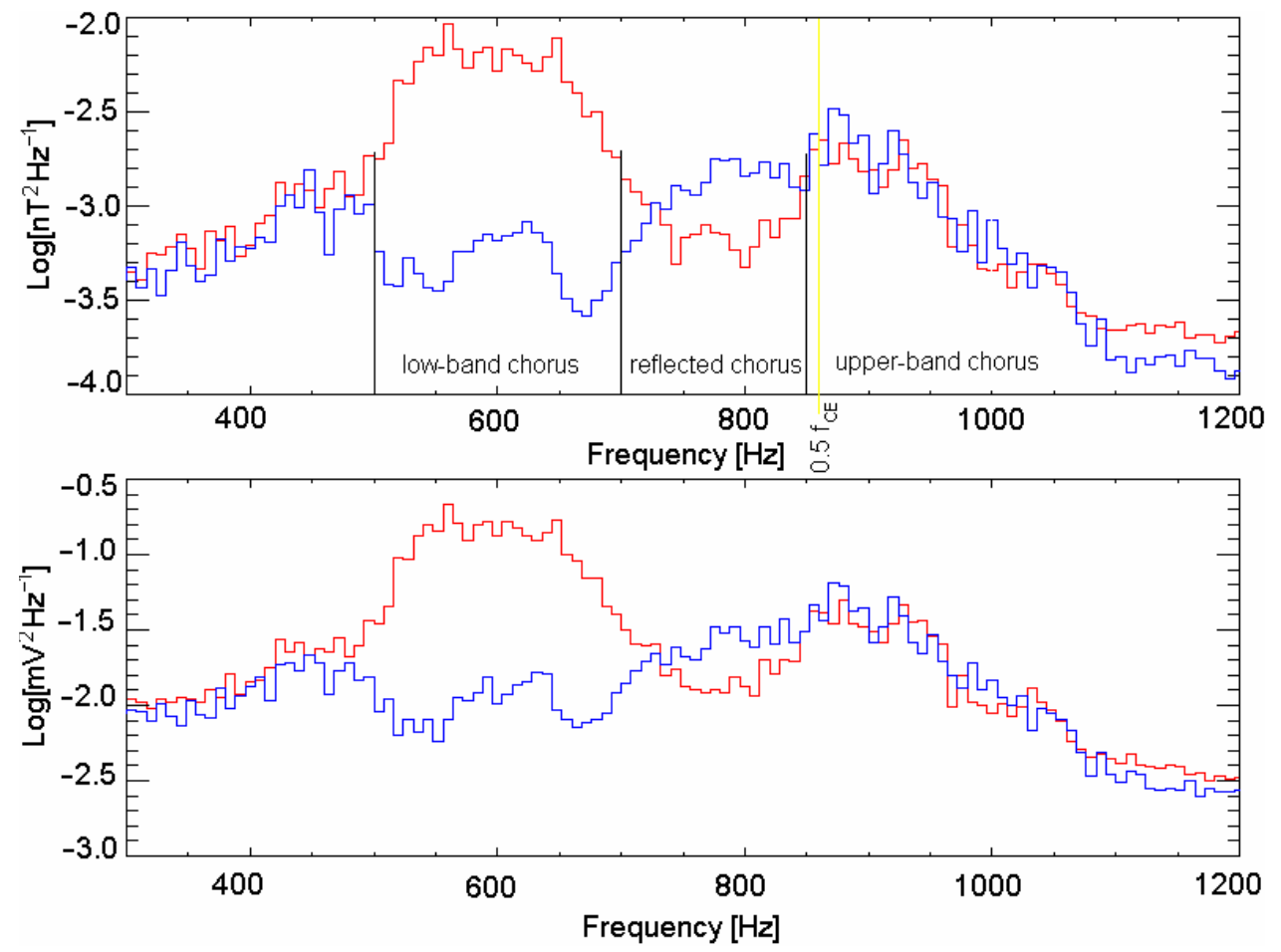

Fig. 5. Magnetic and electric field fluctuation spectra (THA SCW and EFI measurements). Red and blue correspond to times when forward and reflected chorus elements were registered. The estimation of reflected chorus energy gave it nearly 0.01 of the forward chorus energy.

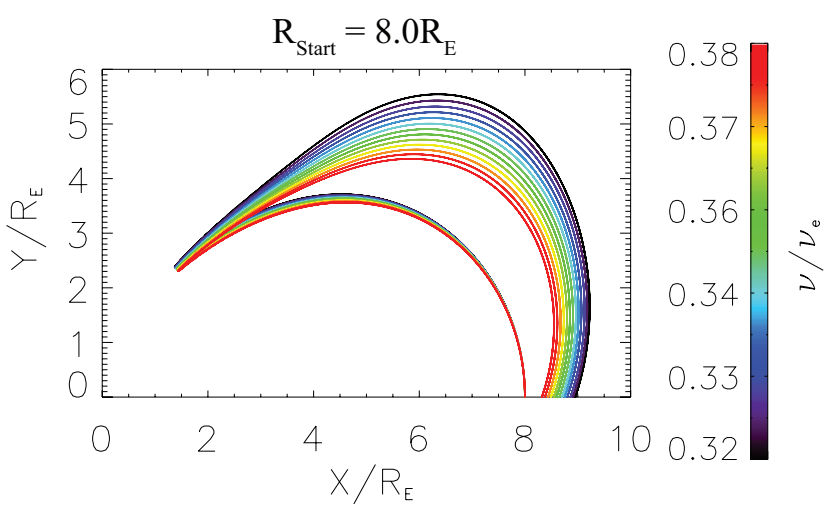

Fig. 6. All whistlers start parallel to the local magnetic field, at $L=8$. After reflection the waves spread towards a range of higher latitudes.

\section{Ray tracing of whistler wave packets}

The geometrical optics approach (WKB approximation) is employed to trace electromagnetic whistler waves in the Earth's magnetosphere. A corresponding set of ordinary differential equations describing the evolution of whistler wave-vector, frequency and propagation trajectory can be found elsewhere (Suchy, 1981). This approach is valid when the characteristic scale of the medium property (density, temperature, distribution function, etc.) variation is larger then the wavelength of the whistler wave. In this paper this condition is satisfied since the wavelength of observed whistler waves lie the range (150-250) km while the scale of the global density and magnetic field changes is about $1 R_{\mathrm{E}}$ and the fluctuation spatial scale is about $300 \mathrm{~km}$ transverse to background magnetic field (Agapitov et al., 2011) and $>1000 \mathrm{~km}$ along it. To compute the plasma dispersion function that enters the equations of geometrical optics, we used an algorithm implemented in WHAMP (Waves in Homogeneous Anisotropic Multi-component Plasma) code by K. Rönnmark (Rönnmark, 1982). Density distributions of magnetospheric plasma particles were calculated from the simplified version of the global core plasma model (Gallagher et al., 2000) neglecting longitudinal density variations (density distributions were therefore effectively twodimensional). The magnetic field was assumed to obey the Olson-Pfitzer model (Olson and Pfitzer, 1974) with a dipole corresponding to the year 2005 epoch. Within WHAMP, the plasma dispersion function is calculated for the very general case of hot plasma with possible background flows and nonmaxwellian particle distributions. However, since the global magnetospheric temperature model has only been developed for a spatially restricted region $\left(10^{3} \div 10^{5}\right) \mathrm{km}$ of altitude, such a model based on Akebono observations was proposed by Kutiev et al. (2002), we assumed that all plasma particles obey Maxwellian distributions with uniform temperatures of $1 \mathrm{eV}$. This approach neglects the change in plasma 

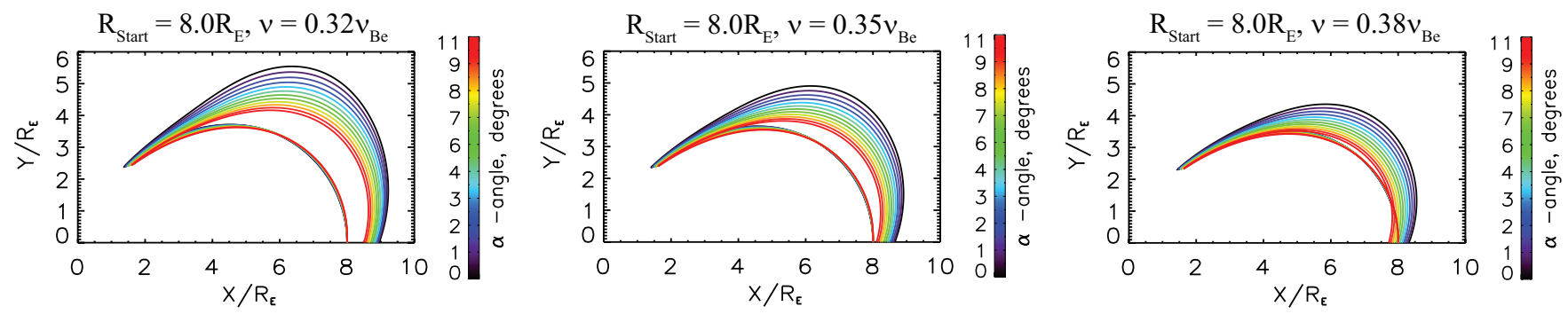

Fig. 7. Whistler ray trajectories for three different wave frequencies. The angle between the magnetic field and whistler's $\boldsymbol{k}$-vector is varied in the range $0^{\circ}-11^{\circ}$. Rays propagate in the meridional plane, $\varphi=0^{\circ}$.
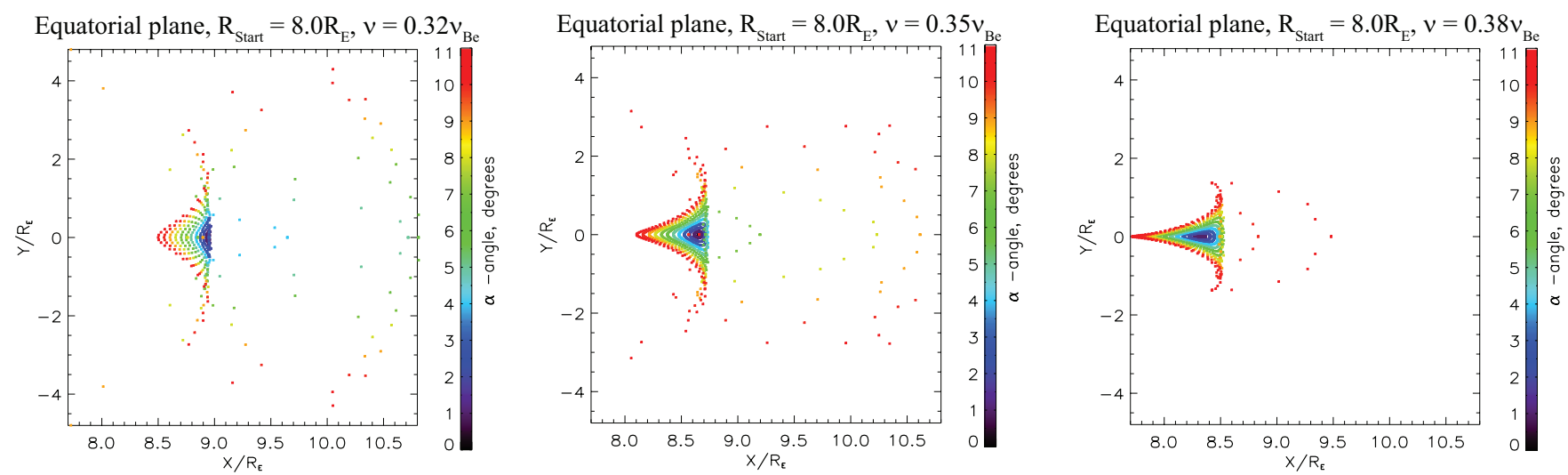

Fig. 8. Color-coded rectangles mark the points at which whistler rays intersect the plane of the magnetic equator after their first low-altitude reflection. Color outlines the initial angle between the wave's $\boldsymbol{k}$-vector and the ambient magnetic field.

temperature along the whistler's trajectory as well as the possibility of resonant wave-particle interaction with energetic non-maxwellian groups of particles.

We suppose that not only in proximity to the registration point, but also in the extended region of equatorial magnetosphere, a mechanism generates whistler chorus in the frequency range $(0.32 \div 0.38) f_{\text {ce }}$. In the observation point $[8.4,0.15,0.42]$ THA recorded simultaneous propagation of the forward and reflected chorus (propagation direction was extracted from the field data by Poynting flux calculation).

When the Olson-Pfitzer magnetic field model is used, the reflected chorus frequencies of $700-800 \mathrm{~Hz}$ correspond to the generation region located at $L \approx 8.0\left(f_{\mathrm{ce}} \approx 2140 \mathrm{~Hz}\right)$. Thus in our simulations we launched electromagnetic whistler waves with different initial frequencies and wave vectors from a point in the plane of magnetic equator with $L=8.0$ and tracked their propagation and low-latitude reflection.

Whistler ray propagation trajectory depends on several parameters - in particular, on wave frequency and the angle between the wave's $\boldsymbol{k}$-vector and the magnetic field. We launched rays with different frequencies within the observed range $685 \mathrm{~Hz}$ to $815 \mathrm{~Hz}$ (which roughly corresponds to $(0.32$ to 0.38$\left.) f_{\mathrm{ce}}\right)$ and registered points in the equatorial plane where these rays return after first reflection. In Figs. 6 and 7 whistler ray trajectories are plotted for different wave frequencies and different angles between $\boldsymbol{k}$ and $\boldsymbol{B}$ at their start points. All the rays in Fig. 6 start parallel to the local magnetic field and differ only by frequency, whereas in Fig. 7, waves are oblique initially (with the angle $\alpha$ between $\boldsymbol{k}$ and $\boldsymbol{B}$ varying in the range $0^{\circ}$ to $11^{\circ}$ ). Trajectories in three subplots are presented for three different frequencies corresponding to the chorus center and edges. For clarity in Figs. 6 and 7 the azimuthal angle $\varphi$ between $\boldsymbol{k}$ and the local meridian plane was forced to zero, thereby ensuring planar ray propagation within the meridional plane of the source. Figure 8 presents results of three-dimensional wave propagation, i.e., without the $\varphi=0$ simplification. Rays with different frequencies and different initial angles between $\boldsymbol{k}$ and $\boldsymbol{B}$ were launched from the magnetic equator position $L=8.0$. Then the points at which rays intersect the plane of magnetic equator after their first low-latitude reflection are plotted by colorized rectangles whose color corresponds to the initial angle between the whistler wave vector and the local magnetospheric magnetic field (angle prescribed at the starting point).

From Figs. 6, 7, and 8 it is evident that the chorus of whistler waves with frequencies in the range $(0.32$ to 0.38) $f_{\text {ce }}$ generated in the vicinity of the magnetic equator at $L=8.0$ will return to the equatorial region after first low-latitude reflection with $L \approx 8.4$ where THA registered 
forward and reflected chorus emissions. From Figs. 7 and 8 it is also evident that the low-frequency chorus sub-band returns to the regions with $L>8.4$ after reflection. Thus, at the point of registration, the reflected whistler chorus spectrum will be truncated from the low-frequency side, which is in excellent agreement with observational data. Qualitative estimations of the spatial energy density of whistler waves after reflection show that the divergence of ray trajectories results in geometrical spreading, and the power of the reflected signal was found to be 20-40 times smaller than the power of the primary signal. This attenuation ratio for each chorus event was obtained in the following way. First, we introduced a uniform spatial grid in a plane region of given space located in the equatorial plane around the point, where particular chorus event was registered. Then, an extended set of whistler rays with their frequencies and wavenumbers covering the entire range of observed chorus signal was launched from each grid point, and the specific wave power of initial signal was introduced as the ratio (total amount of rays)/(size of starting region). After polar reflection, the whistler rays return back to the plane of magnetic equator forming there some pattern with reflected rays distributed non-uniformly within it. Attenuation ratio range was estimated as maximum (or average) value of the specific power of the reflected signal divided by the specific power of the initial signal.

\section{Conclusions}

Electric and magnetic field fluctuations detected aboard THEMIS satellites by SCM and by EFI detectors in the burst mode were used to study magnetospherically-reflected chorus emissions in the outer magnetosphere (L-shell > 7). Chorus waves, which propagate from a well-localized source in the vicinity of the geomagnetic equator to polar regions, can be reflected at lower altitudes. After reflection, wave packets can return to the equator region.

Using waveforms of the electric and magnetic field fluctuations, we determined the direction of the Poynting flux and wave vector distribution for forward and reflected waves. We showed that the reflected chorus emissions observed in the vicinity of the magnetic equator also have a discrete structure roughly similar to that of the chorus emissions propagating poleward; the magnetic and electric field perturbation amplitude of the reflected signal is significantly (20-30 times) smaller, however. Geometrical optics ray-tracing simulations of chorus propagation in the magnetosphere with the realistic model of the geomagnetic field (Olson-Phitzer model) and plasma density distribution (GCPM) were used to explain experimental results. Numerical estimates of the spatial energy density for reflected whistler waves show that because the divergence of ray trajectories results in geometrical spreading, the power of the reflected signal should be 20-40 times smaller than the power of the primary signal. It is worth nothing that observations of ducted whistlers show energy conser- vation for primary and reflected signal with some temporal power spreading due to wave dispersion. Such effects are mostly observed at low $L$-shells $(L \sim 2-3)$; magnetospheric reflection is typically observed for $L>5$.

Reflected emissions observed by particular spacecraft have a frequency shift with respect to the primary chorus that propagates from the magnetic equator. Ray tracing confirms that reflected waves were generated near the geomagnetic equator closer to the Earth where the magnetic field magnitude is larger (L-shell is smaller). They have similar frequency diapason relative to the local value $f_{\text {ce }}$ in the generation region.

Acknowledgements. This work was financially supported by CNES through the grant "Modeles d'ondes", and by the ECO NET program of the EGIDE (France), THEMIS contract NAS5-02099, J. W. Bonnell and F. S. Mozer for use of EFI data, and K. H. Glassmeier, U. Auster and W. Baumjohann for the use of FGM data German Center for Aviation and Space (DLR) under contract 50 OC 0302. Authors thank to Judy Hohl for very useful assistance in preparation of the manuscript.

Guest Editor A. Masson thanks one anonymous referee for her/his help in evaluating this paper.

\section{References}

Agapitov, O., Krasnoselskikh, V., Zaliznyak, Yu., Angelopoulos, V., Le Contel, O., and Rolland, G.: Chorus source region localization in the Earth's outer magnetosphere using THEMIS measurements, Ann. Geophys., 28, 1377-1386, doi:10.5194/angeo28-1377-2010, 2010.

Agapitov O. V., Krasnoselskikh, V., Dudok de Wit, T., Khotyaintsev, Y., Santolik, O., Rolland, G., and Pickett, J. S.: Multi spacecraft Cluster observations of chorus emissions as a tool for the remote sensing of plasma density fluctuations, J. Geophys. Res., in review, 2011.

Anderson, R. R. and Maeda, K.: VLF Emissions Associated With Enhanced Magnetospheric Electrons, J. Geophys. Res., 82(1), 135-146, 1977.

Bonnell, J. W., Mozer, F. S., Delory, G. T., Hull, A. J., Ergun, R. E., Cully, C. M., Angelopoulos, V., and Harvey, P. R.: The Electric Field Instrument (EFI) for THEMIS, Space Sci. Rev., 141, 303341, 2008.

Bortnik, J., Li, W., Thorne, R. M., Angelopoulos, V., Cully, C., Bonnell, J., Le Contel, O., and Roux, A.: An Observation Linking the Origin of Plasmaspheric Hiss to Discrete Chorus Emissions, Science, 324(5928), 775-778, doi:10.1126/science.1171273, 2009.

Burtis, W. J. and Helliwell, R. A.: Banded Chorus - A New Type of VLF Radiation Observed in the Magnetosphere by OGO 1 and OGO 3, J. Geophys. Res., 74(11), 3002-3010, 1969.

Burton, R. K.: Critical Electron Pitch Angle Anisotropy Necessary for Chorus Generation, J. Geophys. Res., 81(25), 4779-4781, 1976.

Burton, R. K. and Holzer, R. E.: The origin and propagation of chorus in the outer magnetosphere, J. Geophys. Res., 79, 1014$1023,1974$. 
Gallagher, D. L., Craven, P. D., and Comfort, R. H.: Global core plasma model, J. Geophys. Res., 105, 18819-18834, doi:10.1029/1999JA000241, 2000.

Goldstein, B. E. and Tsurutani, B. T.: Wave normal directions of chorus near the equatorial source region, J. Geophys. Res., 89, 2789-2810, 1984.

Gurnett, D. and O'Brien, B.: High-Latitude Geophysical Studies with Satellite Injun 3 5. Very-Low-Frequency Electromagnetic Radiation, J. Geophys. Res., 69(1), 65-89, 1964.

Gurnett, D. A., Anderson, R. R., Scarf, F. L., Fredricks, R. W., and Smith, E. J.: Initial results from the ISEE 1 and 2 plasma wave investigation, Space Sci. Rev., 23, 103-122, 1979.

Haque, N., Spasojevic, M., Santoli'k, O., and Inan, U. S.: Wave normal angles of magnetospheric chorus emissions observed on the Polar spacecraft, J. Geophys. Res., 115, A00F07, doi:10.1029/2009JA014717, 2010.

Hayakawa, M., Hattori, K., Lagoutte, D., Parrot, M., and Lefeuvre, F.: Direction finding of chorus emissions in the out magnetosphere and their generation and propagation, Planet.Space Sci., 38, 135-143, 1990.

Helliwell R. A.: Whistlers and related ionospheric phenomena, 1967.

Horne, R. B., Thorne, R. M., Shprits, Y. Y., Meredith, N. P., Glauert, S. A., Smith, A. J., Kanekal, S. G., Baker, D. N., Engebretson, M. J., Posch, J. L., Spasojevic, M., Inan, U. S., Pickett, J. S., and Decreau, P. M. E.: Wave acceleration of electrons in the Van Allen radiation belts, Nature, 437, 227-230, doi:10.1038/nature03939, 2005.

Koons, H. C. and Roeder, J. L.: A survey of equatorial magnetospheric wave activity between 5 and 8 RE, Planet. Space Sci., 38(10), 1335-1341, 1990.

Kutiev, I., Oyama, K., and Abe, T.:, Analytical representation of the plasmasphere electron temperature distribution based on Akebono data, J. Geophys. Res., 10, 1459, doi:10.1029/2002JA009494, 2002.

Le Contel, O., Roux, A., Robert, P., Coillot, C., Bouabdellah, A., de la Porte, B., Alison, D., Ruocco, S., Angelopoulos, V., Bromund, K., Chaston, C. C., Cully, C., Auster, H. U., Glassmeier, K. H. Baumjohann, W., Carlson, C. W., McFadden, J. P., and Larson, D.: The Search Coil Magnetometer for THEMIS, Space Sci. Rev., 141, 0038-6308, 2008.

Li, W., Thorne, R. M., Angelopoulos, V., Bonnell, J. W., McFadden, J. P., Carlson, C. W., LeContel, O., Roux, A., Glassmeier, K. H., and Auster, H. U.: Evaluation of whistler-mode chorus intensification on the nightside during an injection event observed on the THEMIS spacecraft, J. Geophys. Res., 114, A00C14, doi:10.1029/2008JA013554, 2009a.

Li, W., Thorne, R. M., Angelopoulos, V., Bortnik, J., Cully, C. M., Ni, B., Le Contel, O., Roux, A., Auster, U., and Magnes, W.: Global Distribution of Whistler-mode Chorus Waves Observed on the THEMIS Spacecraft, Geophys. Res. Lett., 36, L09104, doi:10.1029/2009GL037595, 2009b

Li, W., Thorne, R., Bortnik, J., Angelopoulos, V., McFadden, J. P., Bonnell, J., Le Contel, O., Roux, A., and Auster, U.: THEMIS analysis of observed equatorial electron distributions responsible for the chorus excitation, J. Geophys. Res., 115, A00F11, doi:10.1029/2009JA014845, 2010.
Means, J. D.: Use of the Three-Dimensional Covariance Matrix in Analyzing the Polarization Properties of Plane Waves, J. Geophys. Res., 77, 5551-5559, 1972.

Meredith, N. P., Horne, R. B., and Anderson, R. R.: Substorm dependence of chorus amplitudes: Implications for the acceleration of electrons to relativistic energies, J. Geophys. Res., 106, 13165-13178, 2001.

Olson, W. P. and Pfitzer, K. A.: A quantitative model of the magnetospheric magnetic field, J. Geophys. Res., 79, 3739-3748, doi:10.1029/JA079i025p03739, 1974.

Omura, Y., Nunn, D., Matsumoto, H., and Rycroft, M. J.: A review of observational, theoretical and numerical studies of VLF triggered emissions, J. Atmos. Terr. Phys., 53, 351-368, 1991.

Parrot, M., Santolík, O., Gurnett, D. A., Pickett, J. S., and Cornilleau-Wehrlin, N.: Characteristics of magnetospherically reflected chorus waves observed by CLUSTER, Ann. Geophys., 22, 2597-2606, doi:10.5194/angeo-22-2597-2004, 2004.

Rönnmark, K.: Waves in homogeneous, anisotropic multicomponent plasmas (WHAMP), Kiruna Geophysical Institute Report No. $179,1982$.

Santolik, O., Parrot, M., and Lefeuvre, F.: Singular value decomposition methods for wave propagation analysis, Radio. Sci., 38(1), 1010, doi:10.1029/2000RS002523, 2003.

Santolik, O., Gurnett, D. A., Pickett, J. S., Parrot, M., and Cornilleau-Wehrlin, N.: Central position of the source region of storm-time chorus, Planet. Space Sci., 53, 299-305, 2005.

Sazhin, S. S. and Hayakawa, M.: Magnetospheric chorus emissions - A review, Planet. Space Sci., 4, 681-697, doi:10.1016/00320633(92)90009-D, 1992.

Shklyar, D. R., Chum, J., and Jiříček, F.: Characteristic properties of $\mathrm{Nu}$ whistlers as inferred from observations and numerical modelling, Ann. Geophys., 22, 3589-3606, doi:10.5194/angeo22-3589-2004, 2004.

Sibeck, D. G. and Angelopoulos, V.: THEMIS science objectives and mission phases, Space Sci. Rev., 141(1-4), 35-59, doi:10.1007/s11214-008-9393-5, 2008

Suchy, K.: Real Hamilton equations of geometric optics for media with moderate absorption, Radio Sci., 16, 1179-1182, doi:10.1029/RS016i006p01179, 1981.

Trakhtengerts, V. Y.: A generation mechanism for chorus emission, Ann. Geophys., 17, 95-100, doi:10.1007/s00585-999-0095-4, 1999.

Tsurutani, B. T. and Smith, E. J.: Postmidnight chorus: a substorm Phenomenon, J. Geophys. Res., 79, 118-127, 1974.

Tsurutani, B. T. and Smith, E. J.: Two Types of Magnetospheric ELF Chorus and Their Substorm Dependences, J. Geophys. Res., 82(32), 5112-5128, 1977.

Yagitani, S., Nagano, I., Matsumoto, H., Omura, Y., Paterson, W. R., Frank, L. A., and Anderson, R. R.: Wave and particle measurements for chorus emissions by GEOTAIL in the magnetosphere, Adv. Space Res., 24, 91-94, 1999. 